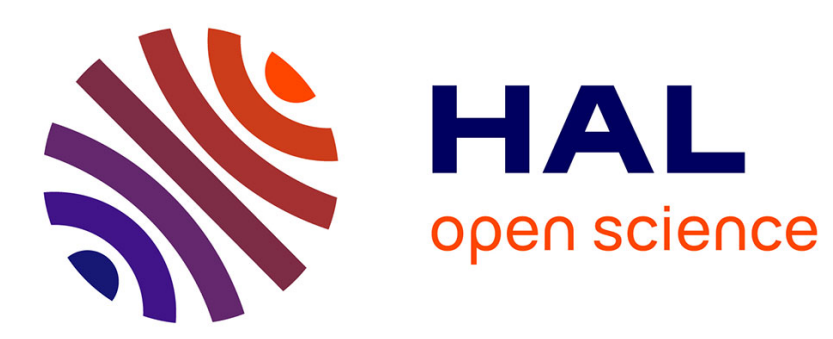

\title{
Extension of CSRSM for the parametric study of the face stability of pressurized tunnels
}

\author{
Guilhem Mollon, Daniel Dias, Abdul-Hamid Soubra
}

\section{To cite this version:}

Guilhem Mollon, Daniel Dias, Abdul-Hamid Soubra. Extension of CSRSM for the parametric study of the face stability of pressurized tunnels. Georisk 2011, Geotechnical Risk Assessment and Management, ASCE, 2011, Atlanta, United States. 10.1061/41183(418)126 . hal-01008213

\section{HAL Id: hal-01008213 \\ https://hal.science/hal-01008213}

Submitted on 21 Nov 2018

HAL is a multi-disciplinary open access archive for the deposit and dissemination of scientific research documents, whether they are published or not. The documents may come from teaching and research institutions in France or abroad, or from public or private research centers.
L'archive ouverte pluridisciplinaire HAL, est destinée au dépôt et à la diffusion de documents scientifiques de niveau recherche, publiés ou non, émanant des établissements d'enseignement et de recherche français ou étrangers, des laboratoires publics ou privés. 


\title{
Extension of CSRSM for the Parametric Study of the Face Stability of Pressurized Tunnels
}

\author{
Guilhem Mollon ${ }^{1}$, Daniel Dias ${ }^{2}$, and Abdul-Hamid Soubra ${ }^{3}$, M.ASCE
}

${ }^{1}$ LGCIE, INSA Lyon, Université de Lyon, Domaine scientifique de la Doua, Bât JCA Coulomb, 20 Avenue Albert Einstein, 69621 Villeurbanne, France; guilhem.mollon@insa-lyon.fr

${ }^{2}$ LGCIE, INSA Lyon, Université de Lyon, Domaine scientifique de la Doua, Bât JCA Coulomb, 20 Avenue Albert Einstein, 69621 Villeurbanne, France ; daniel.dias@insa-lyon.fr

${ }^{3}$ GeM, University of Nantes, Boulevard de l'université, BP 152, 44603 Saint-Nazaire cedex, France ; abed.soubra@univ-nantes.fr

\begin{abstract}
The Collocation-based Stochastic Response Surface Methodology (CSRSM) is a powerful probabilistic method. It aims at replacing a complex deterministic model by a simple analytical expression (called meta-model) to reduce the time cost of the classical probabilistic methods. The meta-model is based on a Polynomial Chaos Expansion (PCE). The coefficients of the PCE are computed in this paper by regression from the response of the deterministic model at a limited number of collocation points. The conventional formalism of CSRSM requires performing a new set of deterministic computations each time the probabilistic parameters of the input random variables are slightly modified. An extension of CSRSM is therefore proposed in this paper. It allows the realization of a parametric study at a limited time cost without loss of accuracy. This is demonstrated by comparing the results obtained from the original CSRSM and its extension when performing a parametric study concerning the stability analysis of a pressurized tunnel face.
\end{abstract}

\section{INTRODUCTION}

As is well known, the most robust probabilistic approach is the Monte-Carlo simulation method. This method requires a large number of calls of the deterministic model especially for small values of the failure probability (e.g. about 1,000,000 samples for a failure probability of $10^{-5}$ ).

Although the deterministic numerical methods (such as those based on the Finite Element Method FEM or the Finite Difference Method FDM) are appealing, these methods are difficult to use in a probabilistic framework particularly when employing MC simulation method. This is because these methods need a very long computation time. In order to overcome the above shortcoming, the Collocationbased Stochastic Response Surface Methodology (CSRSM) is applied herein. CSRSM makes possible the probabilistic study of a complex deterministic model by replacing it by an analytical expression (called meta-model) with a reduced time cost. This meta-model is expressed by a Polynomial Chaos Expansion (PCE). The coefficients of the PCE are determined in this paper by a regression approach using a limited number of calls of the deterministic model. The meta-model can then be used in the classical probabilistic methods such as the Monte Carlo simulation method. It should be mentioned that the conventional formalism of CSRSM requires performing a new set of deterministic computations each time the probabilistic parameters of the 
input random variables are slightly modified. An extension of CSRSM is therefore proposed in this paper. It allows the realization of a parametric study at a limited time cost without loss of accuracy. As an illustrative application of CSRSM and its extension, one considers in this paper the probabilistic stability analysis of a pressurized tunnel face. The paper is organised as follows: A brief description of the deterministic model concerning the collapse pressure of a pressurized tunnel face is first presented. It is followed by the presentation of CSRSM and its extension. Finally, a verification of CSRSM (by comparison with the results of Monte Carlo simulation method) and a comparison between the results obtained from both CSRSM and its extension are presented and discussed.

\section{DETERMINISTIC MODEL}

The face stability analysis of a circular tunnel driven by a pressurized shield is of major interest. It requires the determination of a so-called "critical collapse pressure" of the tunnel face (denoted $\sigma_{\mathrm{c}}$ ), i.e. the greatest one under which the tunnel face collapses. The deterministic model chosen in this study is analytical. It is based on the kinematical theorem of limit analysis. The collapse mechanism is translational. It is composed of five conical blocks (Fig. 1) with an opening angle equal to $\varphi$ where $\varphi$ is the soil friction angle.

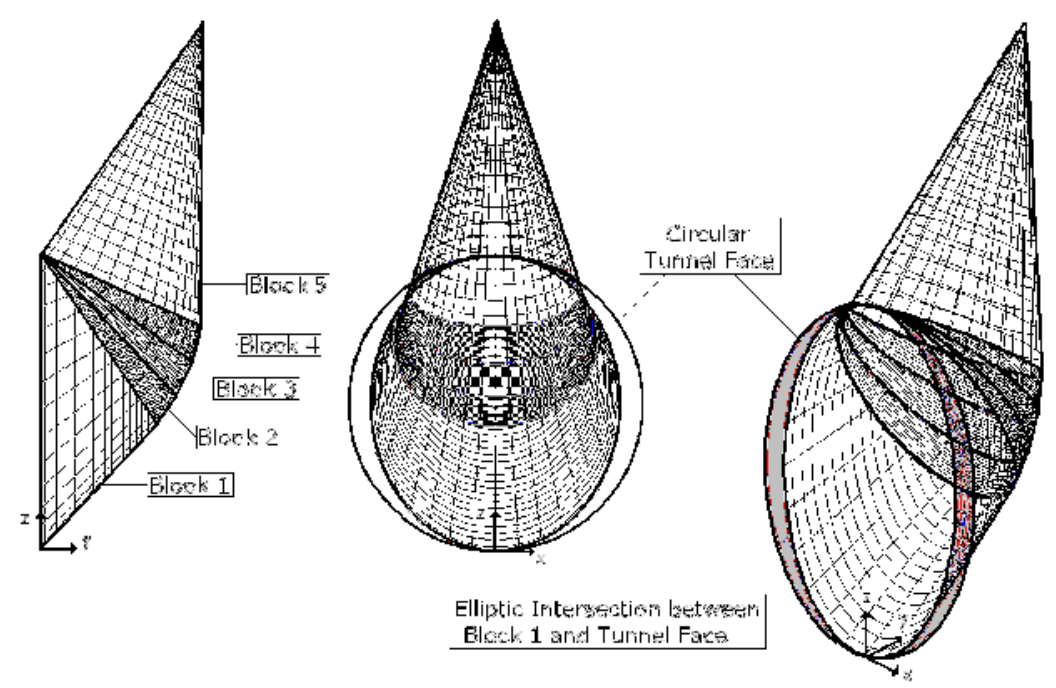

Figure 1. Five-block collapse mechanism

This mechanism was extensively presented in Mollon et al. [2009]: it is able to provide a quite correct estimation of $\sigma_{\mathrm{c}}$ despite the fact that it does not intersect the entire circular tunnel face as was observed experimentally by Takano et al. (2006). The analytical five-block model was chosen in this study because its extremely small time cost (smaller than $0.1 \mathrm{~s}$ ) makes it very convenient for the verification of CSRSM. This verification is performed by comparison with Monte Carlo simulation method which requires a great number of calls of the deterministic model. In this study, the output random variable is the critical collapse pressure, and the two input random variables are the friction angle $(\varphi)$ and the cohesion (c) of the soil. 


\section{BRIEF DESCRIPTION OF CSRSM}

In this section, the classical formalism of CSRSM (Isukapalli [1999], Sudret [2007], Phoon and Huang [2007] and Huang et al. [2009]) is presented in the case of two input random variables ( $\varphi$ and $c)$. CSRSM aims at replacing the initial deterministic model by an approximate analytical expression (meta-model) when performing a probabilistic analysis. The meta-model is a Polynomial Chaos Expansion (PCE). For a PCE of order $n$, the meta-model is expressed in the basis of the multidimensional Hermite polynomials of order $\leq n$. For a given set of the probabilistic parameters of the input random variables (i.e. type of distribution and statistical moments of the random variables, and correlations between random variables); the unknown coefficients of the PCE are obtained in this paper by regression using the response of the deterministic model at a given number of collocation points. The different steps of CSRSM may be described as follows:

The two random variables (i.e. $\varphi$ and c) have first to be represented in the PCE by two standard variables $\xi_{1}$ and $\xi_{2}$, which are normal uncorrelated variables with zero mean and unit variance. For a PCE of order $n$, the available collocation points are classically determined as follows: each standard variable is assumed to take the values of the roots of the one-dimensional Hermite polynomial of order $n+1$, and the available collocation points result from the combinations of all these roots for the different random variables. It should be noted that this method of choosing the collocation points is not mandatory as will be seen later in this paper. For the present case of two input random variables, the output random variable may be expressed by a PCE as follows:

$U=\sum_{i=1}^{p} a_{i} \cdot \Gamma_{i}\left(\xi_{1}, \xi_{2}\right)$

In this expression, the terms $\Gamma_{\mathrm{i}}$ are multidimensional Hermite polynomials (which constitute the basis of the PCE) and the terms $a_{i}$ are unknown coefficients to be determined. The number $p$ of terms in this summation and the number $M$ of the available collocation points are given by:

$p=\frac{\left(n+n_{v}\right) !}{n ! n_{v} !}$

$M=(n+1)^{n_{v}}+\left\{\begin{array}{cc}0 & \text { if one of the roots of the }(\mathrm{n}+1)^{\text {th }} \text { univariate Hermite polynomial is } 0 \\ 1 & \text { otherwise }\end{array}\right.$

where $\mathrm{n}_{\mathrm{v}}$ is the number of random variables. It should be mentioned that when the number $\mathrm{n}_{\mathrm{v}}$ of the random variables is important, the number $\mathrm{M}$ of the available collocation points becomes much larger than the number $\mathrm{p}$ of the unknown coefficients. This leads to a linear system of equations where the number of equations is greater than the number of unknown. It is therefore necessary to make a judicious choice of the collocation points to be used in the regression process. Several methods exist (Isukapalli [1999], Sudret [2007]), but they are useless when using only two random variables as is the case in the present paper. Hence, all the available collocation points are considered in the analysis. To be introduced in the deterministic 
model, the collocation points represented by vectors $\left(\xi_{1, m}, \xi_{2, m}\right)$ (with $1 \leq m \leq M$ ) have to be expressed in the space of the physical variables corresponding to vectors $\left(\varphi_{m}, c_{m}\right)$, using the following expressions:

$$
\begin{gathered}
{\left[\begin{array}{l}
\xi_{1 C, m} \\
\xi_{2 C, m}
\end{array}\right]=H \cdot\left[\begin{array}{l}
\xi_{1, m} \\
\xi_{2, m}
\end{array}\right]} \\
\left\{\begin{array}{l}
\varphi_{m}=F_{\varphi}^{-1}\left[\Phi\left(\xi_{1 C, m}\right)\right] \\
c_{m}=F_{c}^{-1}\left[\Phi\left(\xi_{2 C, m}\right)\right]
\end{array}\right.
\end{gathered}
$$

In these equations, $\mathrm{H}$ is the Cholesky transform of the correlation matrix of $\varphi$ and $\mathrm{c}, \mathrm{F}_{\varphi}$ is the cumulative density function (CDF) of $\varphi, \mathrm{F}_{\mathrm{c}}$ is the $\mathrm{CDF}$ of $\mathrm{c}$, and $\Phi$ is the normal cumulative density function with zero mean and unit variance. When the response of the deterministic model (i.e. the value of $\sigma_{c}$ ) is obtained for each collocation point, the values of the PCE coefficients are determined by regression using the following equation:

$\underline{\underline{N^{t}}} \cdot \underline{\underline{N}} \cdot \underline{a}=\underline{\underline{N^{t}}} \cdot \underline{f}$

where $a=$ column vector of the unknown coefficients, $f=$ column vector of the deterministic responses, and $\mathrm{N}$ is a matrix given by:

$$
\underline{\underline{N}}=\left[\begin{array}{cccccc}
\Gamma_{0,0} & \Gamma_{1,0}\left(\xi_{1,1}\right) & \Gamma_{0,1}\left(\xi_{2,1}\right) & \Gamma_{2,0}\left(\xi_{1,1}\right) & \Gamma_{1,1}\left(\xi_{1,1}, \xi_{2,1}\right) & \Gamma_{0,2}\left(\xi_{2,1}\right) \\
\Gamma_{0,0} & \Gamma_{1,0}\left(\xi_{1,2}\right) & \Gamma_{0,1}\left(\xi_{2,2}\right) & \Gamma_{2,0}\left(\xi_{1,2}\right) & \Gamma_{1,1}\left(\xi_{1,2}, \xi_{2,2}\right) & \Gamma_{0,2}\left(\xi_{2,2}\right) \\
\cdot & \cdot & \cdot & \cdot & \cdot & \cdot \\
\Gamma_{0,0} & \Gamma_{1,0}\left(\xi_{1, M}\right) & \Gamma_{0,1}\left(\xi_{2, M}\right) & \Gamma_{2,0}\left(\xi_{1, M}\right) & \Gamma_{1,1}\left(\xi_{1, M}, \xi_{2, M}\right) & \Gamma_{0,2}\left(\xi_{2, M}\right)
\end{array}\right]
$$

Once the unknown coefficients are determined, one obtains an analytical equation (i.e. a meta-model) that can be used in place of the deterministic model to perform a probabilistic analysis using (for example) Monte Carlo simulation method.

\section{VERIFICATION OF CSRSM}

A set of probabilistic parameters is chosen as a reference case for the two input random variables $\varphi$ and $\mathrm{c}$ as follows: the two variables are considered as normal uncorrelated variables with $\mu_{\varphi}=17^{\circ}$ and $\operatorname{COV}(\varphi)=10 \%, \mu_{\mathrm{c}}=7 \mathrm{kPa}$ and $\operatorname{COV}(\mathrm{c})=20 \%$. Characteristics of a soft clay are chosen because this kind of soil is likely to allow face instabilities. For this reference case, the coefficients of the PCEs of orders 2 to 5 are determined using the process described above. Fig. 2 shows the response surfaces (represented by lines of equal value of $\sigma_{\mathrm{c}}$ in a $\varphi$-c plane) as provided by the original deterministic model and by its approximations by the PCEs of orders 2 and 4 . It clearly appears that both PCEs are able to provide a very good approximation of the original deterministic model in the central part (i.e. in the region of maximum probability of occurrence), but that the order 4 is much more accurate in the surrounding areas corresponding to zones with lower probabilities of occurrence. 
A Monte-Carlo (MC) simulation with $10^{6}$ samples is performed on the original deterministic model and on the PCEs of orders 2 to 5 . This simulation is only made possible due to the very small time cost of the original deterministic model chosen in this paper, which allows a great number of samples. The results in terms of probability density function (PDF) and in terms of failure probability are shown in Figs. $3 a$ and $3 b$ respectively. It appears from Fig. $3 a$ that the PDF obtained for the meta-models are in good agreement with the one of the original deterministic model, especially when the PCE order is larger than 2. Moreover, Fig. $3 \mathrm{~b}$ shows that all the PCEs of order greater than 2 provide a good estimation of the failure probabilities at the distribution tail which is the zone of interest of the geotechnical engineer; the accuracy increases with the PCE order.
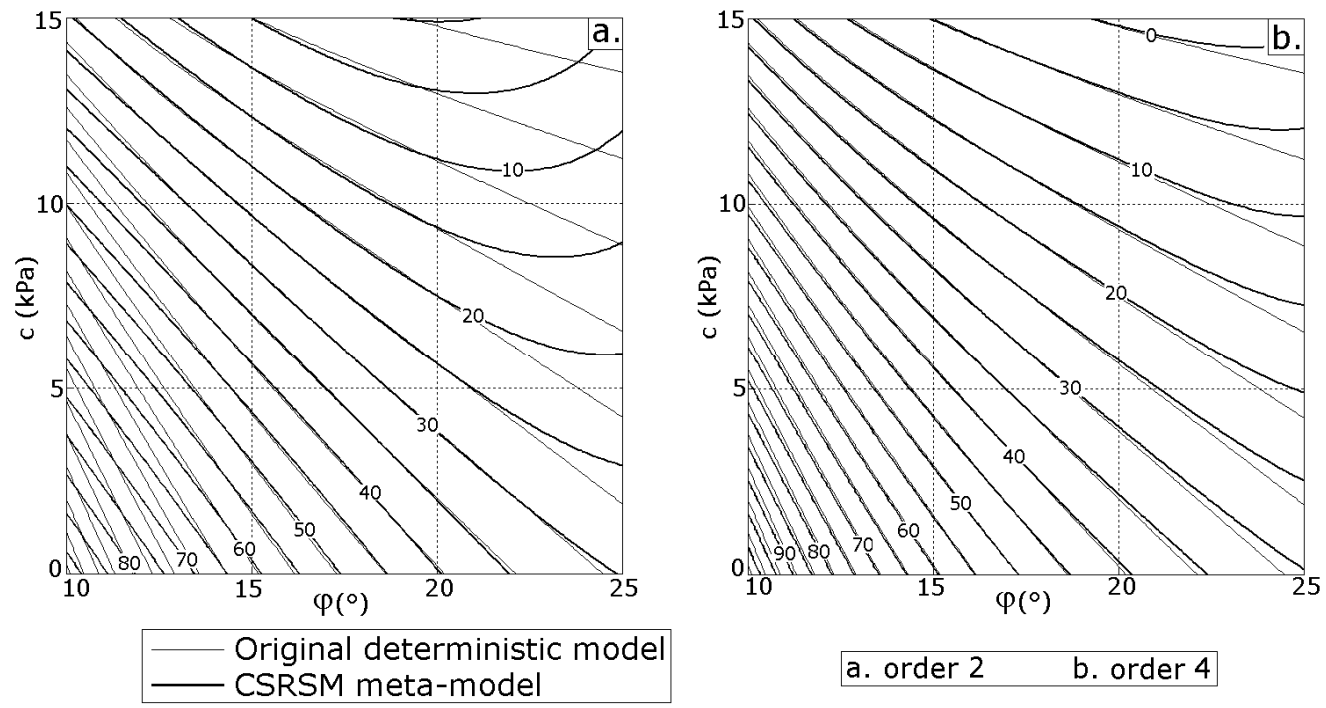

Figure 2. Response surfaces of the critical collapse pressure (in $\mathrm{kPa}$ ) as provided by the original deterministic model and by the PCEs of orders 2 and 4
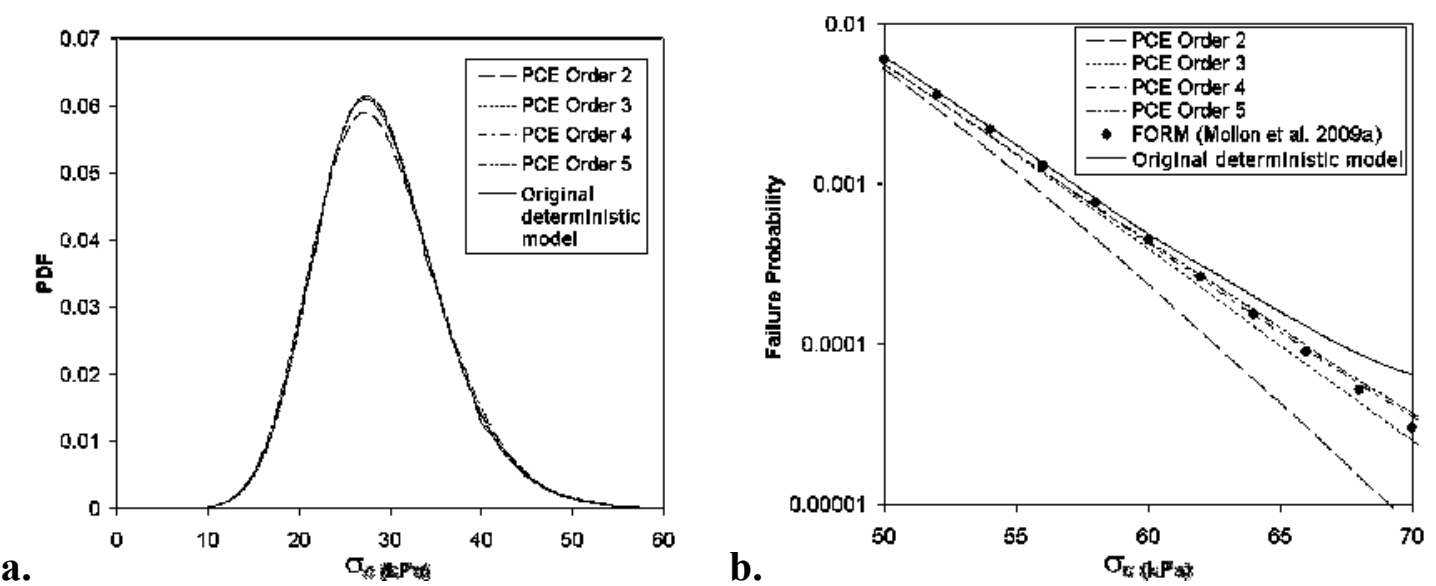

Figure 3. Results of the Monte-Carlo Simulation; a. PDF of $\sigma_{c} ; \mathbf{b}$. Failure probability

As a conclusion, the PCE of order 2 provides good results only in terms of trends, but the PCEs of orders 3, 4 and 5 provide very close probabilities to the ones of the original deterministic model. There is also a good agreement with FORM results obtained in a previous study (Mollon et al. [2009]). The PCE of order 4 seems 
to be the best compromise between accuracy and computational cost, but the order 3 may reasonably be used for computationally-expensive models. Notice finally that the number of collocation points used for the fourth order PCE is equal to 25 , which means that 25 calls of the deterministic model were needed to obtain this PCE.

\section{EXTENSION OF CSRSM}

CSRSM is an efficient and accurate tool for the probabilistic study of a mechanical model, but it suffers from the fact that its conventional formalism implies a new set of deterministic computations each time one of the probabilistic parameters of the input variables (such as the COV or the correlation coefficient) is changed. This is due to equations (4) and (5) which state that the position of the collocation points in the physical space is dependant on the correlation matrix and the CDF of the input variables. The time cost of a parametric study (when using a computationallyexpensive model) is therefore very expensive, while such a study may be interesting because these parameters are usually not known with a great accuracy. This section presents an extension of CSRSM, which makes possible the use of existing deterministic results to carry on a parametric study with no additional time cost. This extension is based on the fact that the position of the collocation points described earlier is not mandatory and that it is possible to use other kinds of collocation schemes, without loss of accuracy of the meta-model. For example, Fig. 4a shows two response surfaces of $\sigma_{\mathrm{c}}$ in the physical $\varphi$-c plane. The first one corresponds to a PCE of order 4 used for the reference case described above, and the second one corresponds to a PCE of order 4 for a different probabilistic case (lognormal variables with $\mu_{\varphi}=17^{\circ}$ and $\operatorname{COV}(\varphi)=8 \%, \mu_{\mathrm{c}}=7 \mathrm{kPa}$ and $\operatorname{COV}(\mathrm{c})=25 \%$ and with a correlation coefficient $\left.\rho_{\varphi c}=-0.3\right)$. Since the type of the probability distributions, the COVs and the correlation coefficient have been changed, the collocation points are different with respect to the reference case. However, the two response surfaces appear very similar on the entire range of the parameters. This illustrates the claim that there is no need for new calls of the deterministic model for a parametric study. When a first set of deterministic computations has been performed and a new set of probabilistic parameters is chosen, one may compute the positions of the collocation points in the standard space which would have led to the physical collocation points that were already computed. These positions $\left(\xi_{1, m}^{\prime}, \xi_{2, m}^{\prime}\right)$ are obtained by the following equations:

$$
\begin{gathered}
\left\{\begin{array}{l}
\xi_{1 C, m}^{\prime}=\Phi^{-1}\left(F_{\varphi}\left(\varphi_{m}\right)\right) \\
\xi_{2 C, m}^{\prime}=\Phi^{-1}\left(F_{c}\left(c_{m}\right)\right)
\end{array}\right. \\
{\left[\begin{array}{l}
\xi_{1, m}^{\prime} \\
\xi_{2, m}^{\prime}
\end{array}\right]=H^{-1}\left[\begin{array}{l}
\xi_{1 C, m}^{\prime} \\
\xi_{2 C, m}^{\prime}
\end{array}\right]}
\end{gathered}
$$

This process provides a new set of collocation points $\left(\xi_{1, m}^{\prime}, \xi_{2, m}^{\prime}\right)$ for which the corresponding deterministic results are already available. Equation (6) can therefore be applied to compute the coefficients of a new PCE, with no additional deterministic computation. A MC simulation with $10^{6}$ samples is carried out for the new probabilistic properties described above, using the three following models: (i) the original deterministic model, (ii) the PCE of order 4 based on the conventional 
CSRSM (which implies 25 new calls to the deterministic model), and (iii) the PCE of order 4 based on the proposed extension method (which uses only the existing 25 deterministic results and do not require any new call to the deterministic model).
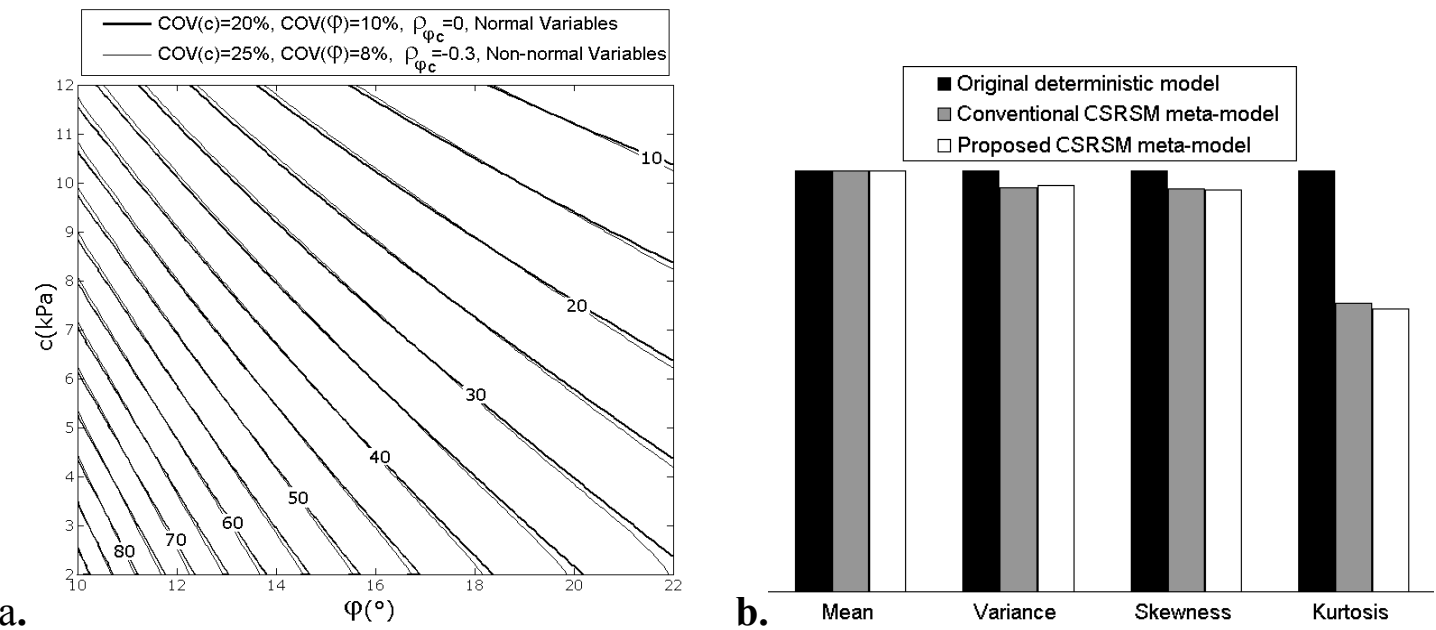

Figure 4. a. comparison between the response surfaces provided by two PCEs with different probabilistic parameters; $b$. Statistical moments of the conventional and proposed CSRSM (normalized to the ones of the original deterministic model)
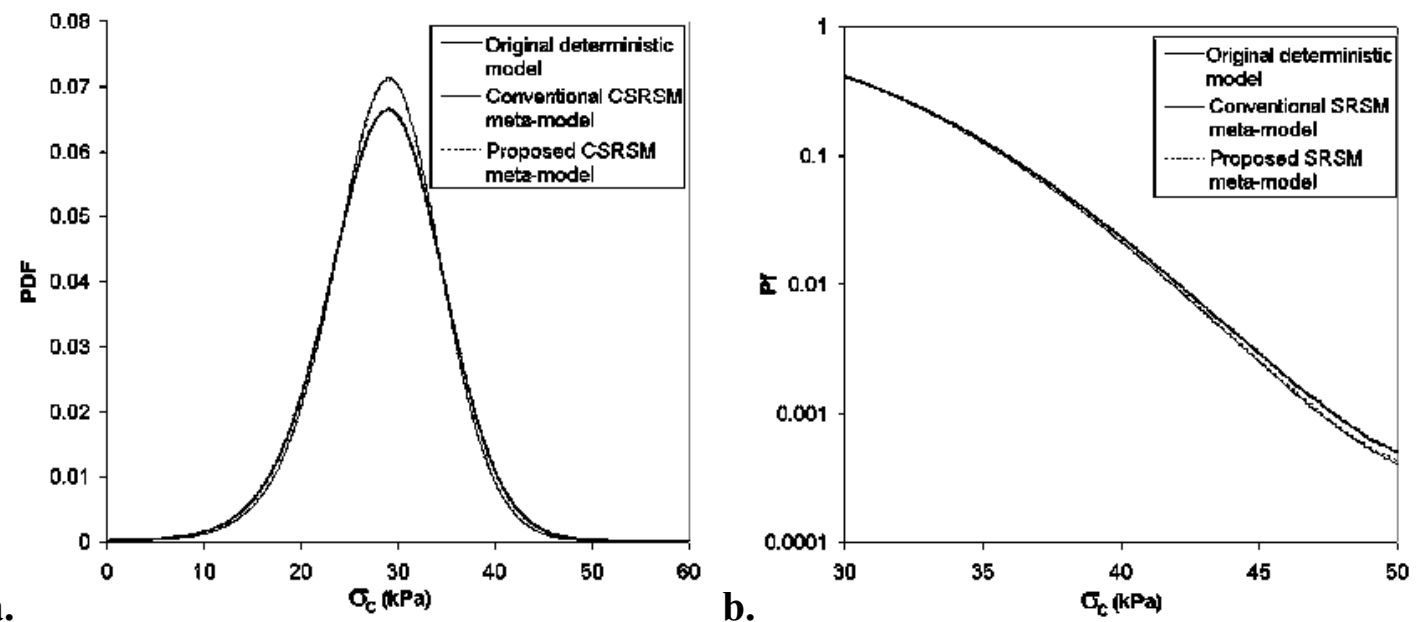

Figure 5. Comparison of the classical and the proposed CSRSM with respect to the original deterministic model; a. PDF of $\sigma_{c} ; b$. Failure probability

Figs. $4 \mathrm{~b}, 5 \mathrm{a}$ and $5 \mathrm{~b}$ present a comparison between the three models, respectively in terms of the statistical moments of $\sigma_{\mathrm{c}}$, in terms of the PDF of $\sigma_{\mathrm{c}}$, and in terms of the probability of failure at the distribution tail. A very good agreement appears between the two meta-models. It clearly means that the proposed extension of CSRSM does not reduce the accuracy of the probabilistic results. Notice however that the proposed methodology is only relevant if the area covered by the new collocation points in the standard space is not too different from the one obtained using the original collocation scheme. From a practical point of view, it means that the changes made to the probabilistic parameters of the input variables should remain small during the parametric study. 


\section{CONCLUSION}

The Collocation-based Stochastic Response Surface Methodology (CSRSM) is presented and applied to an analytical deterministic model (devoted to the determination of the critical collapse pressure of a pressurized tunnel face). CSRSM is first verified by a comparison with the results obtained from the Monte-Carlo (MC) simulation method. In a second time, an extension of the CSRSM is proposed, allowing a parametric study for no additional call to the deterministic model. The proposed extension method is verified by a MC simulation approach. It shows that the proposed collocation scheme does not reduce the accuracy of the meta-model. However, the proposed methodology is only relevant if the area covered by the new collocation points in the standard space is not too different from the one obtained using the original collocation scheme. From a practical point of view, it means that the changes made to the probabilistic parameters of the input variables should remain small during the parametric study.

\section{REFERENCES}

Huang, S.P., Liang, B., Phoon, K.K. (2009). "Geotechnical probabilistic analysis by collocation-based stochastic response surface method." Georisk, 3(2), 75-86.

Isukapalli, S.S. (1999). An uncertainty analysis of transport-transformation models, Ph.D. thesis, The State University of New Jersey, New Brunswick, New Jersey.

Mollon, G., Dias, D., and Soubra, A.-H. (2009). "Probabilistic analysis and design of circular tunnels against face stability." Int. J. of Geomech., ASCE, 9(6), 237249.

Phoon, K.K., and Huang, S.P. (2007). "Geotechnical probabilistic analysis using collocation-based stochastic response surface method.", Applications of Statistics and Probability in Civil Engineering, Kanda, Takada and Furada (eds.), Taylor and Francis Group, London.

Sudret, B. (2007). "Global sensitivity analysis using polynomial chaos expansion.", Reliability Engineering and System Safety, 93, 964-979.

Takano, D., Otani, J., Nagatani, H., Mukunoki, T. (2006). “Application of X-ray CT on boundary value problems in geotechnical engineering - Research on tunnel face failure." Proceedings of Geocongress, ASCE, Atlanta. 